\title{
Selective spatial enhancement: Attentional spotlight size impacts spatial but not temporal perception
}

\author{
Stephanie C. Goodhew ${ }^{1} \cdot$ Elizabeth Shen $^{1} \cdot$ Mark Edwards $^{1}$
}

Published online: 3 May 2016

(C) Her Majesty the Queen in Right of Australia 2015

\begin{abstract}
An important but often neglected aspect of attention is how changes in the attentional spotlight size impact perception. The zoom-lens model predicts that a small ("focal") attentional spotlight enhances all aspects of perception relative to a larger ("diffuse" spotlight). However, based on the physiological properties of the two major classes of visual cells (magnocellular and parvocellular neurons) we predicted trade-offs in spatial and temporal acuity as a function of spotlight size. Contrary to both of these accounts, however, across two experiments we found that attentional spotlight size affected spatial acuity, such that spatial acuity was enhanced for a focal relative to a diffuse spotlight, whereas the same modulations in spotlight size had no impact on temporal acuity. This likely reflects the function of attention: to induce the high spatial resolution of the fovea in periphery, where spatial resolution is poor but temporal resolution is good. It is adaptive, therefore, for the attentional spotlight to enhance spatial acuity, whereas enhancing temporal acuity does not confer the same benefit.
\end{abstract}

Keywords Attention - Spatial attention - Spotlight .

Zoom-lens $\cdot$ Spatial $\cdot$ Temporal $\cdot$ Magnocellular $\cdot$

Parvocellular $\cdot$ Visual perception

Spatial attention is a core visual mechanism that selects certain aspects of a visual scene for preferential processing while minimizing the processing of others. This selection prevents

Stephanie C. Goodhew

stephanie.goodhew@anu.edu.au

1 Research School of Psychology, The Australian National University, Building 39, Canberra, ACT 0200, Australia the visual system from being overwhelmed by the sheer volume of information available at a given point in time. The prevailing metaphor for spatial attention is that of a spotlight, where stimuli inside the spotlight are selected for privileged processing at the expense of stimuli outside of the spotlight (e.g., Eriksen \& Hoffman, 1973; Grubert, Righi, \& Eimer, 2013; Tsal \& Lavie, 1988). One aspect of this attentional spotlight that has been extensively studied is the impact of covertly shifting the location of the spotlight on perception (known as transient attention). Typically it has been found that moving the attentional spotlight to a location improves perceptual processing at that location, as evidenced by enhanced detection of stimulus onset (Posner, 1980; Posner, Snyder, \& Davidson, 1980), enhanced character, letter, or word target identification (Eriksen \& Hoffman, 1972; Folk, Remington, \& Johnston, 1992; Goodhew, Kendall, Ferber, \& Pratt, 2014; Prinzmetal, Presti, \& Posner, 1986; Schreij, Owens, \& Theeuwes, 2008; Yantis \& Jonides, 1984), improved orientation discrimination (Baldassi \& Burr, 2000; Morgan, Ward, \& Castet, 1998), and improved texture segmentation in the periphery (Yeshurun \& Carrasco, 1998).

The effect of cueing the attentional spotlight to a given location, however, is not universally beneficial. Texture segmentation is impaired in central vision (Yeshurun \& Carrasco, 1998), and temporal resolution, as measured by temporal gap detection and discrimination of direction of apparent motion identification, is impaired (Yeshurun \& Hein, 2011; Yeshurun \& Levy, 2003), and perceived duration is prolonged (Enns, Brehaut, \& Shore, 1999; Yeshurun \& Marom, 2008). This led Yeshurun and colleagues to conclude that cueing transient attention to a location leads to a processing bias characteristic of parvocellular neurons (visual cells that have small receptive fields, and superior spatial acuity but poor temporal acuity and prolonged response persistence relative to magnocellular neurons (Denison, Vu, Yacoub, Feinberg, \& Silver, 2014; 
Derrington \& Lennie, 1984; Livingstone \& Hubel, 1988)), and a concurrent suppression of magnocellular neurons.

This dissociation between magnocellular- and parvocellular-mediated perceptual performance may be important in informing our understanding of another aspect of visual attention: spotlight size. That is, a critically important but often neglected aspect of attention is the size of the attentional spotlight, in the absence of a shift of attention (a form of sustained rather than transient attention). There are instances where a number of factors, including the nature of visual stimuli or task demands, compel spatial-attentional resources to be spread more broadly across the visual field, such as the requirement to integrate multiple spatially-disperse aspects of a scene, or uncertainty about target location. This had led to the "zoom-lens" extension to the spotlight model of attention, according to which the system can trade off the size of the attended area for precision of encoding, much like a camera lens (Eriksen \& St. James, 1986; Eriksen \& Yeh, 1985). That is, when attentional resources are focussed in a small region ("focal" spotlight), then the concentration of these resources is said to lead to a greater facilitation of perceptual performance compared with when these resources are spread more thinly over a larger area ("diffuse" spotlight). Consistent with this prediction, responses to identify and detect letters and characters are improved (Eriksen \& St. James, 1986; Eriksen \& Yeh, 1985; LaBerge, 1983), visual search for letters is facilitated (Greenwood \& Parasuraman, 1999), and functional magnetic resonance imaging (fMRI)- measured activation in early visual areas is increased (Muller, Bartelt, Donner, Villringer, \& Brandt, 2003), to a greater extent with a focal relative to a diffuse attentional spotlight. Notably, however, all of these behavioral measures gauge spatial acuity only, and the fMRI study measured activation of cortical regions which receive both parvocellular and magnocellular inputs, where the task was to detect colored shapes and therefore presumably predominately a parvocellular-mediated process. In other words, according to the zoom-lens model, all aspects of perceptual performance are enhanced with a focal spotlight, yet the testing of this account has been limited to tasks that are predominately parvocellular-mediated.

Given the differential impact of transient attention on magnocellular- and parvocellular-mediated perceptual tasks, it is possible that a similar dissociation occurs in modulations of spotlight size in the absence of a shift of spotlight location. In fact, the properties of parvocellular and magnocellular neurons provide a clear mediating mechanism for instantiating different spotlight sizes. That is, parvocellular neurons have smaller receptive fields, ideal for instantiating a focal spotlight, whereas magnocellular neurons have larger receptive field sizes, ideal for implementing a diffuse spotlight. This, therefore, leads us to propose a novel theory for the effect of spotlight size on perception: the spatiotemporal trade-off account. According to this account, instantiating a focal spotlight calls preferentially upon parvocellular-neuron input, which produces enhanced spatial acuity but impaired temporal acuity. A diffuse spotlight, in contrast, requires the upregulation of magnocellular input, thereby enhancing temporal acuity at the cost of spatial acuity. Note that both the zoomlens model and the spatiotemporal trade-off account make the same prediction for the impact of spotlight size on spatial acuity: improved performance for focal relative to diffuse. Where they differ, however, is the impact on temporal acuity. The zoom-lens model predicts that a focal spotlight improves all aspects of perception, including temporal acuity, whereas according to the spatiotemporal trade-off account, performance depends on the match between the nature of the stimulus and the properties of the different cells, such that temporal acuity will be impaired with a focal relative to a diffuse spotlight. The purpose of the present study, therefore, was to pit the differing predictions from these two theoretical accounts against one another, by measuring the impact of modulations in spotlight size on temporal acuity, in addition to spatial acuity.

\section{Experiment 1}

The purpose of Experiment 1 was to pit the differing predictions regarding the impact of spotlight size on temporal acuity arising from the zoom-lens model and the spatiotemporalaccount directly against one another. The zoom-lens model predicts enhanced temporal acuity for a focal relative to diffuse spotlight, whereas the spatiotemporal trade-off account predicts impaired temporal acuity for a focal relative to a diffuse spotlight. Both accounts predict enhanced spatial acuity for a focal relative to a diffuse spotlight. This means that the zoom-lens model predicts a consistent advantage for both perceptual acuity modalities for a focal spotlight relative to a diffuse spotlight, whereas the spatiotemporal trade-off account predicts a disordinal interaction between spotlight size and perceptual acuity modality, such that spatial acuity is improved for focal versus diffuse, whereas temporal acuity is improved for diffuse relative to focal.

In order to distinguish between these possibilities, in the present study observers performed two tasks, one was designed to induce a small or large attentional spotlight size, and the other was to measure their spatial or temporal acuity. The induction task was a two-alternative forced-choice which required observers to identify the shape (circle or slightly oval) of a small (focal inducer) or a large (diffuse inducer) stimulus, a task which they performed on $80 \%$ of experimental trials. Inducer type was blocked, meaning that observers completed all the diffuse or all of the focal trials consecutively (order counterbalanced across observers). The small stimulus was designed to narrow observers' attentional spotlight, whereas the large stimulus was intended to expand observers' 
attentional spotlight size. Then, in order to measure the impact of this spotlight size induction on spatial and temporal acuity, on the other $20 \%$ of trials, observers performed spatial and a temporal gap detection tasks (varied across block, order counterbalanced). Note that these spatial and temporal gap detection tasks have previously been successfully used to gauge M-cell and P-cell input to perception and how this is affected by various factors, including covert shifts of attention (Yeshurun \& Levy, 2003), the proximity of the hands to visual stimuli (Bush \& Vecera, 2014; Gozli, West, \& Pratt, 2012), and the emotional content of observed facial expressions (Bocanegra \& Zeelenberg, 2011).

\section{Method}

Participants Forty-one participants (34 female) were recruited and participated in exchange for course credit or pay. Their mean age was 20.6 years $(\mathrm{SD}=3.2)$, and four of them were left-handed. All reported normal or corrected-to-normal vision and provided written informed consent prior to participation.

Stimuli and apparatus Stimuli were presented on a cathoderay tube (CRT) gamma-corrected monitor running at a $75-\mathrm{Hz}$ refresh rate. Viewing distance was fixed at $44 \mathrm{~cm}$ with a chinrest. Stimuli were programmed in Matlab using the Psychophysics Toolbox (Brainard, 1997). The background was set to gray $\left(63 \mathrm{~cd} / \mathrm{m}^{2}\right)$. Inducer stimuli were outline circles or ellipses, with a line width of about $0.2^{\circ}$ of visual angle. Focal inducers had a diameter of $1^{\circ}$ when it was a circle and dimensions horizontal $=1^{\circ}$, vertical $=0.8^{\circ}$ when it was an ellipse, whereas diffuse inducers had a diameter of $20^{\circ}$ when it was a circle and dimensions horizontal $=20^{\circ}$, vertical $=16^{\circ}$ when it was an ellipse. For the gap detection blocks, stimuli were outline discs of $0.4^{\circ}$ diameter ( $.08^{\circ}$ line width). In the spatial gap detection block, the gray target was either an unbroken disc, or had a small gap in the bottom $\left(\right.$ size $\left.=.04^{\circ}\right)$. In the temporal gap detection block, the target stimuli were always black unbroken discs. All stimuli presented were centred on the centre of the screen.

Design The experiment was a 2 (inducer type) $\times 2$ (gap detection task) repeated-measures design, where gap detection accuracy was the dependent measure.

Procedure The experiment consisted of two types of trials, inducer task trials and gap detection trials. From the participants' point of view, the primary task was to identify whether the inducer was a circle or an ellipse (inducer task trial). The inducer was dark gray and was presented for $53 \mathrm{~ms}$ before the screen was blank until a response was registered. These inducer trials occurred on $80 \%$ of trials. The purpose of this inducer task was to manipulate attentional spotlight size (focal or diffuse). Inducer type was blocked (order counterbalanced) to maximize the effectiveness of the manipulation (i.e., sustained attention). The remaining $20 \%$ of trials were the gap detection trials, which provided our acuity measures of interest. In one block, the designated task was spatial gap detection, which required participants to determine whether there was a small spatial gap present in the disc or not, and in the other block the designated task was temporal gap detection, which required participants to determine whether there was a single, continuous disc, or two presentations of the disc separated by a brief blank interval (see Fig. 1). In the spatial gap detection block, in order to ensure that accuracy was not at ceiling, the gap detection stimuli were presented at reduced contrast (darker gray), than the temporal gap detection stimuli which were black. Responses for both the inducer and gap detection tasks were via designated keys on the keyboard ("Z" and "?" keys).

Prior to each experimental block, participants completed a practice block of 20 trials which provided feedback on the accuracy of their responses. If necessary, participants repeated this practice block until they scored a minimum of $75 \%$ correct before progressing to the experimental block. The experiment consisted of 480 total trials, 240 per gap detection block. Of these, 48 were gap detection trials, and 192 were inducer trials per block.

\section{Results and discussion}

Participants were excluded from the analysis if they failed to achieve a minimum of $60 \%$ accuracy (where chance level performance is $50 \%$ ) on the inducer task within each gap detection condition. This was done to ensure that we only analysed data from participants who were willing and able to perform the inducer task and thus we can be confident that their attentional

\section{Spatial Gap Detection:}

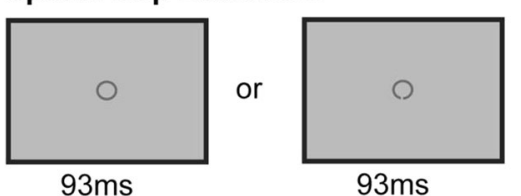

Temporal Gap Detection:

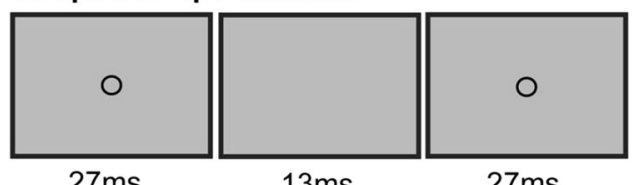

$27 \mathrm{~ms}$

$13 \mathrm{~ms}$

$27 \mathrm{~ms}$

or

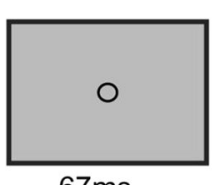

$67 \mathrm{~ms}$

Fig. 1 Schematic representation of the possible stimuli for the spatial and temporal gap detection tasks 
spotlight size was effectively modulated by the task. Participants were also excluded from the analysis if they did not reach a minimum of $60 \%$ accuracy on the gap detection task with at least one type of inducer. This was intended to eliminate cases where the participant was unwilling or unable to perform the gap detection task appropriately. Five datasets were excluded from the analysis according to the inducer criterion, three according to the gap detection accuracy criterion, and another one met both of these criteria. One participant's data were excluded because they only completed one of the two blocks, and one other dataset was replaced when the file was corrupted. The following analyses were for the remaining 30 datasets.

Inducer task accuracy Inducer task accuracy was high ( $>91 \%$ ) for all conditions (see Table 1 for specific values). This demonstrates that these participants were actively engaged in the inducer identification task, and therefore we assume that the size of their attentional spotlight would modulate to the size required to complete the task (small for focal, large for diffuse).

Gap detection accuracy A repeated-measures ANOVA revealed that neither the main effect of gap detection task, $F(1$, $29)=2.08, p=.160, \eta_{\mathrm{p}}{ }^{2}=.067$, nor the main effect of inducer type $(F<1)$, were significant. The interaction between gap detection task and inducer type, however, was significant, $F(1$, 29) $=6.70, p=.015, \eta_{\mathrm{p}}{ }^{2}=.188$ (Fig. 2).

Planned comparisons were used to follow up this interaction, which revealed that for the spatial gap detection task, mean accuracy was significantly greater with the focal inducer $(\mathrm{M}=78.2 \%)$ than with the diffuse inducer $(\mathrm{M}=71.7 \%)$, $t(29)=3.09, p=.004$. Importantly, this suggests that the inducer identification task successfully modulated attentional spotlight size. For the temporal gap detection, while numerically there was the opposite pattern, such that the diffuse inducer condition $(\mathrm{M}=79.9 \%)$ produced greater accuracy than the focal inducer condition $(\mathrm{M}=75.9 \%)$, this difference was not statistically reliable, $t(29)=-1.34, p=.189$.

Altogether then, as both models predicted, and as is consistent with existing evidence, spatial acuity was improved with a focal relative to a diffuse spotlight. Yet contrary to both the zoom-lens model and the spatiotemporal trade-off account there was no reliable impact of spotlight size on temporal acuity. This means that spotlight size was selectively modulating spatial acuity. Before we interpret the theoretical

Table 1 Accuracies (\% correct) for the inducer task as a function of inducer type and gap detection condition

\begin{tabular}{lll}
\hline & Focal inducer & Diffuse inducer \\
\hline Spatial gap detection & 91.7 & 96.3 \\
Temporal gap detection & 91.4 & 97.0 \\
\hline
\end{tabular}

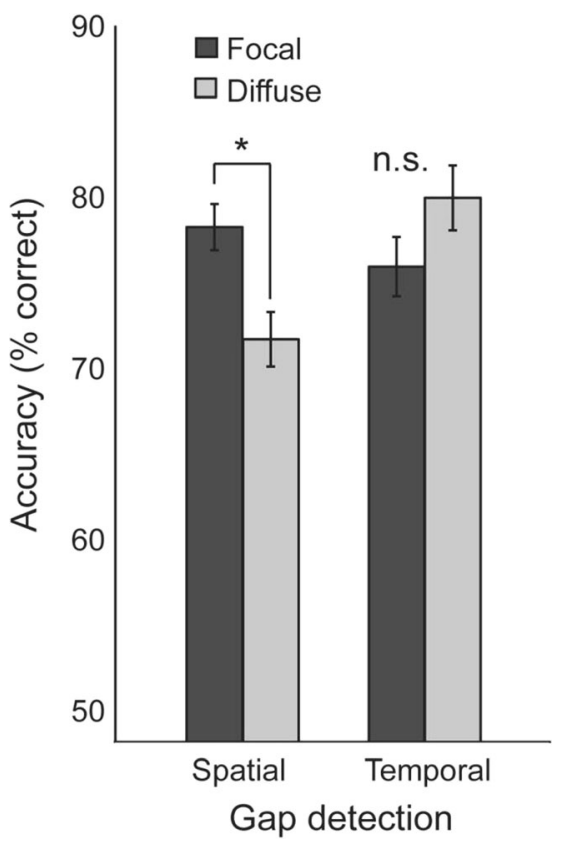

Fig. 2 Schematic representation of gap detection accuracy (\% correct) for each gap detection type (spatial or temporal) as a function of each attentional spotlight size (focal or diffuse). Error bars depict standard errors with Cousineau's (2005) correction for within-subjects designs

significance of this result, however, it is important to test a methodological explanation for the absence of an effect of spotlight size on performance in temporal gap detection task. That is, the total duration of the stimuli in the temporal gap detection task was shorter $(67 \mathrm{~ms})$ than the spatial gap detection task (93 ms), introducing, for instance, differential opportunities for temporal integration and perceived brightness of the stimuli. To ensure that this difference was not responsible for the pattern of results of observed, we conducted Experiment 2, in which the duration of the temporal gap detection stimuli was boosted to $93 \mathrm{~ms}$.

\section{Experiment 2}

Here the total duration of the temporal gap detection stimuli was increased to $93 \mathrm{~ms}$, such that it was on par with the duration of the spatial gap detection stimuli in Experiment 1. If the induction of focal versus diffuse spotlight sizes once again has no reliable impact on temporal gap detection performance, then we can be confident that this reflects a true dissociation between spatial and temporal acuity in the impact of attentional spotlight size.

\section{Method}

Participants Forty individuals (23 female) participated; their mean age was 21.2 years $(\mathrm{SD}=2.5)$, and two reported being left-handed and two ambidextrous. 
Stimuli, apparatus, and procedure These were identical to Experiment 1, with the following exceptions. The experiment consisted exclusively of temporal gap detection trials, of which there were 960 . Temporal gap detection stimuli were presented for either a continuous $93 \mathrm{~ms}$, or $40 \mathrm{~ms}$, followed by a 13-ms temporal gap, and then a further $40 \mathrm{~ms}$ (total duration $=93 \mathrm{~ms}$ ). The luminance of the test stimulus was reduced to $43.5 \mathrm{~cd} / \mathrm{m}^{2}$ in order to offset the improved accuracy observed in pilot testing produced by increasing the duration of the temporal gap stimulus.

\section{Results and discussion}

Data from two participants were excluded from the analysis because they failed to achieve a minimum of $60 \%$ accuracy in both inducer conditions, data from another five were excluded for failing to achieve a minimum of $60 \%$ accuracy in at least one of the gap detection conditions, and two met both of these exclusion criteria. The inducer task accuracy for the remaining participants was high, with an average accuracy of $93.4 \%$ for the focal condition and $97.8 \%$ for the diffuse condition.

Temporal gap detection accuracy for the focal ( $\mathrm{M}=80.3 \%)$ versus diffuse (77.2\%) attentional spotlight conditions was then submitted to a repeated-measures $t$-test. This revealed no effect of spotlight condition, $t(30)=1.69, p=.101$. Altogether then, temporal acuity, as measured by temporal gap detection, was once again not impacted by changes in spotlight size, even with stimulus presentation durations akin to those used in the spatial gap detection in Experiment 1, where accuracy was modulated by spotlight size.

\section{General discussion}

Across two experiments we obtained evidence that taskinduced sustained changes in the size of the attentional spotlight modulated spatial acuity, such that spatial acuity improved with a focal relative to a diffuse spotlight, but had no impact on temporal acuity. This is consistent with neither the zoom-lens model, which predicts an enhancement for both spatial and temporal acuity relative for focal relative to diffuse, nor the spatiotemporal trade-off account, which predicts improved spatial but impaired temporal acuity for a focal relative to a diffuse spotlight. This indicates that a new theoretical account of the impact of spotlight size on perception is required. The finding that attention selectively impacts spatial resolution makes functional sense. It has been suggested that the purpose of covert attention is to simulate the highresolution fovea in the periphery (Carrasco, 2011), where spatial resolution is low, but temporal acuity is high. From this perspective, it is logical that attention should only modulate spatial acuity. Of course, in the present study, stimuli were all centered on fixation and thus covert and overt attention were aligned. It is likely, however, that the mechanisms of attention are optimized for peripheral perception where covert and overt operations diverge.

We see two plausible candidate neurophysiological mechanisms for this dissociation between spatial and temporal acuity in terms of the impact of spotlight size, which may operate either alone or in concert. The first is the possibility modulations in spotlight size alter the input of parvocellular neurons only, with no active suppression of magnocellular neurons. This selectivity has been implicated in relation to the impact of shifting the attentional spotlight on perception (Srinivasan \& Brown, 2006). Although modulations of attentional spotlight size do reflect a different process to shifting the attentional spotlight, they may have a common underlying physiological basis in that parvocellular neurons play a pivotal role. The second possibility is that since shifting attention converges together the centers of multiple receptive fields to the attended area (e.g., Womelsdorf, Anton-Erxleben, Pieper, \& Treue, 2006), then this may be how different spotlight sizes are instantiated. Such a mechanism is likely to predominately affect spatial acuity. In other words, a focal spotlight concentrates the receptive fields of neurons in a very small area, resulting in very high density input and therefore excellent spatial resolution, whereas a diffuse spotlight spreads the receptive fields over a larger area, resulting in a lower density input and therefore a weaker spatial acuity enhancement.

In conclusion, changing the size of the attentional spotlight affects spatial but not temporal acuity. This could reflect attentional effects being selective to modulations of parvocellular input, and/or the convergence of receptive fields exclusively affecting spatial resolution. Future research could benefit from further investigating whether attentional spotlight size can be transiently modulated (see Castiello \& Umiltà, 1990; Yeshurun \& Carrasco, 2008), and the impact of this on different aspects of perception.

Acknowledgments This research was supported by an Australian Research Council (ARC) Discovery Early Career Researcher Award (DE140101734) awarded to S.C.G. and an ARC Discovery Grant (DP110104553) awarded to M.E. The authors thank Reuben Rideaux for assistance with the data collection.

\section{References}

Baldassi, S., \& Burr, D. C. (2000). Feature-based integration of orientation signals in visual search. Vision Research, 40(10-12), 12931300. doi: $10.1016 / \mathrm{S} 0042-6989 \% 2800 \% 2900029-8$

Bocanegra, B. R., \& Zeelenberg, R. (2011). Emotion-induced trade-offs in spatiotemporal vision. Journal of Experimental Psychology: General, 140(2), 272-282. doi:10.1037/a0023188

Brainard, D. H. (1997). The psychophysics toolbox. Spatial Vision, 10(4), 433-436. doi:10.1163/156856897X00357 
Bush, W. S., \& Vecera, S. P. (2014). Differential effect of one versus two hands on visual processing. Cognition, 133(1), 232-237. doi:10. 1016/j.cognition.2014.06.014

Carrasco, M. (2011). Visual attention: The past 25 years. Vision Research, 51(13), 1484-1525. doi:10.1016/j.visres.2011.04.012

Castiello, U., \& Umiltà, C. (1990). Size of the attentional focus and efficiency of processing. Acta Psychologica, 73(3), 195-209. doi: 10.1016/0001-6918(90)90022-8

Cousineau, D. (2005). Confidence intervals in within-subject designs: A simpler solution to Loftus and Masson's method. Tutorial in Quantitative Methods for Psychology, 1(1), 42-45.

Denison, R. N., Vu, A. T., Yacoub, E., Feinberg, D. A., \& Silver, M. A. (2014). Functional mapping of the magnocellular and parvocellular subdivisions of human LGN. NeuroImage, 2, 358-369. doi:10. 1016/j.neuroimage.2014.07.019

Derrington, A. M., \& Lennie, P. (1984). Spatial and temporal contrast sensitivities of neurones in the lateral geniculate nucleus of the macaque. Journal of Physiology, 357, 219-240.

Enns, J. T., Brehaut, J. C., \& Shore, D. I. (1999). The duration of a brief event in the mind's eye. Journal of General Psychology, 126(4), 355-372. doi:10.1080/00221309909595371

Eriksen, C. W., \& Hoffman, J. E. (1972). Temporal and spatial characteristics of selective encoding from visual displays. Perception \& Psychophysics, 12(2-B), 201-204. doi:10.3758/BF03212870

Eriksen, C. W., \& Hoffman, J. E. (1973). The extent of processing of noise elements during selective encoding visual displays. Perception \& Psychophysics, 14, 155-160.

Eriksen, C. W., \& St. James, J. D. (1986). Visual attention within and around the field of focal attention: A zoom lens model. Perception \& Psychophysics, 40(4), 225-240. doi:10.3758/BF03211502

Eriksen, C. W., \& Yeh, Y.-Y. (1985). Allocation of attention in the visual field. Journal of Experimental Psychology: Human Perception and Performance, 11(5), 583-597. doi:10.1037/0096-1523.11.5.583

Folk, C. L., Remington, R. W., \& Johnston, J. C. (1992). Involuntary covert orienting is contingent on attentional control settings. Journal of Experimental Psychology: Human Perception and Performance, 18(4), 1030-1044. doi:10.1037/0096-1523.18.4.1030

Goodhew, S. C., Kendall, W., Ferber, S., \& Pratt, J. (2014). Setting semantics: Conceptual set can determine the physical properties that capture attention. Attention, Perception, \& Psychophysics, 76(6), 1577-1589. doi:10.3758/s13414-014-0686-3

Gozli, D. G., West, G. L., \& Pratt, J. (2012). Hand position alters vision by biasing processing through different visual pathways. Cognition, 124(2), 244-250. doi:10.1016/j.cognition.2012.04.008

Greenwood, P., \& Parasuraman, R. (1999). Scale of attentional focus in visual search. Perception \& Psychophysics, 61(5), 837-859. doi:10. 3758/BF03206901

Grubert, A., Righi, L. L., \& Eimer, M. (2013). A unitary focus of spatial attention during attentional capture: Evidence from event-related brain potentials. Journal of Vision, 13(3), 9.

LaBerge, D. (1983). Spatial extent of attention to letters and words. Journal of Experimental Psychology: Human Perception and Performance, 9(3), 371-379. doi:10.1037/0096-1523.9.3.371
Livingstone, M., \& Hubel, D. (1988). Segregation of form, color, movement, and depth: Anatomy, physiology, and perception. Science, 240(4853), 740-749. doi:10.1126/science.3283936

Morgan, M., Ward, R., \& Castet, E. (1998). Visual search for a tilted target: Tests of spatial uncertainty models. The Quarterly Journal of Experimental Psychology A: Human Experimental Psychology, 51A(2), 347-370. doi:10.1080/027249898391666

Muller, N. G., Bartelt, O. A., Donner, T. H., Villringer, A., \& Brandt, S. A. (2003). A physiological correlate of the "zoom lens" of visual attention. Journal of Neuroscience, 23(9), 3561-3565.

Posner, M. I. (1980). Orienting of attention. The Quarterly Journal of Experimental Psychology, 32(1), 3-25. doi:10.1080/ 00335558008248231

Posner, M. I., Snyder, C. R. R., \& Davidson, B. J. (1980). Attention and the detection of signals. Journal of Experimental Psychology: General, 109(2), 160-174. doi:10. 1037/0096-3445.109.2.160

Prinzmetal, W., Presti, D. E., \& Posner, M. I. (1986). Does attention affect visual feature integration? Journal of Experimental Psychology: Human Perception and Performance, 12(3), 361-369. doi:10. 1037/0096-1523.12.3.361

Schreij, D., Owens, C., \& Theeuwes, J. (2008). Abrupt onsets capture attention independent of top-down control settings. Perception \& Psychophysics, 70(2), 208-218. doi:10.3758/PP.70.2.208

Srinivasan, N., \& Brown, J. M. (2006). Effects of endogenous spatial attention on the detection and discrimination of spatial frequencies. Perception, 35(2), 193-200. doi:10.1068/p5314

Tsal, Y., \& Lavie, N. (1988). Attending to color and shape: The special role of location in selective visual processing. Perception \& Psychophysics, 44(1), 15-21. doi:10.3758/bf03207469

Womelsdorf, T., Anton-Erxleben, K., Pieper, F., \& Treue, S. (2006). Dynamic shifts of visual receptive fields in cortical area MT by spatial attention. Nature Neuroscience, 9(9), 1156-1160. doi:10. $1038 / \mathrm{nn} 1748$

Yantis, S., \& Jonides, J. (1984). Abrupt visual onsets and selective attention: Evidence from visual search. Journal of Experimental Psychology: Human Perception and Performance, 10(5), 601621. doi:10.1037/0096-1523.10.5.601

Yeshurun, Y., \& Carrasco, M. (1998). Attention improves or impairs visual performance by enhancing spatial resolution. Nature, 396(6706), 72-75. doi:10.1038/23936

Yeshurun, Y., \& Carrasco, M. (2008). The effects of transient attention on spatial resolution and the size of the attentional cue. Perception \& Psychophysics, 70(1), 104-113. doi:10.3758/PP.70.1.104

Yeshurun, Y., \& Hein, E. (2011). Transient attention degrades perceived apparent motion. Perception, 40(8), 905-918. doi:10.1068/p7016

Yeshurun, Y., \& Levy, L. (2003). Transient spatial attention degrades temporal resolution. Psychological Science, 14(3), 225-231. doi: 10.1111/1467-9280.02436

Yeshurun, Y., \& Marom, G. (2008). Transient spatial attention and the perceived duration of brief visual events. Visual Cognition, 16(6), 826-848. doi:10.1080/13506280701588022 